\title{
Impact of MDR1 genetic polymorphisms on postoperative piritramide analgesia
}

\author{
Matouskova $\mathrm{O}^{1}$, Slanar $\mathrm{O}^{1}$, Adamkova $\mathrm{J}^{2}$, Pafko $\mathrm{P}^{2}$, Perlik $\mathrm{F}^{1}$, Adamek $\mathrm{S}^{2}$ \\ Institute of Pharmacology, First Faculty of Medicine, General Teaching Hospital, Charles University in Prague, \\ Czech Republic. sadamek@post.cz
}

\begin{abstract}
Objectives: The aim of prospective study was to evaluate the therapeutic efficacy of piritramide in patients after removal of parathyroid glands in relation to MDR1 genotype. In the treatment of moderate acute postoperative pain, piritramide plays a major role. It is difficult to predict its optimal therapeutic efficacy and tolerability in individual patients.

Methods: We compared the effect of piritramide in 56 patients after surgical removal of parathyroid glands in a prospective study. We evaluated pain intensity, pain difference and sum of pain difference (SPID) using visual analogue scale (VAS in $\mathrm{mm}$ ) and adverse effects in the relationship with the MDR1 - polymorphism of G2677T/A. Results: In the wild-type group (2677GG), there was maximal pain difference of $30.6 \pm 24.9$ and SPID of 209.33 \pm 95.80 while in genotype 2677TT and 2677GT, the corresponding values were $19.5 \pm 25.5$ and $147.07 \pm 91.38$, respectively. In group of patients with wild type of $2677 \mathrm{GG}$ genotype, there was $80 \%$ of responders with more than $50 \%$ reduction in VAS as compared to baseline while in group with carriers of 2677T allele, there are only $39 \%$ of responders present $\left(X^{2}=5.83 ; p=0.016\right)$. Furthermore, the total consumption of piritramide was lower in comparison with the variant-allele carrying group $(p=0.008)$. The total incidence of adverse drug reactions was observed in $40 \%$ of patients with wild type of $2677 \mathrm{GG}$ genotype when compared to $83 \%$ in the group carrying the variant allele $\left(X^{2}=7.92 ; p=0.005\right)$. Significantly more patients in the wild-type group were satisfied with postoperative pain treatment in comparison to the variant allele group $\left(X^{2}=6.49 ; p=0.0109\right)$. Conclusion: We observed a better analgesic effect of piritramide and a decreased incidence of side effects in the wild-type genotype (2677GG) group, when compared with variant-allele carrying patients (Tab. 2, Fig. 1, Ref. 7). Full Text in PDF www.elis.sk.

Key words: analgesia, piritramide, postoperative pain, adverse effects, polymorphism of MDR1.
\end{abstract}

\begin{abstract}
Abbreviations: MDR1 - Multidrug resistence 1, VAS - Visual analogue scale, DNA - Deoxyribonucleic acid, PCR - RFLP Polymerase chain reaction - restriction fragment length polymorphism, SPID - sum of pain intensity difference
\end{abstract}

Piritramide is a drug of choice for the treatment of moderate to severe postoperative pain. Acute pain represents an important factor significantly reducing the quality of life in postoperative period. This may lead to numerous unfavourable consequences including increased risk of delirium, depression, sleep disorders and higher risk of mortality. Central analgesic effects of piritramide are comparable to those of morphine, but the inter-individual variability is high. Approximately one third of the patients suffer from insufficient alleviation from pain. Optimal therapeutic efficacy of

${ }^{1}$ Institute of Pharmacology, First Faculty of Medicine, General Teaching Hospital, Charles University in Prague, Czech Republic, and ${ }^{2}$ Third Department of Surgery, First Faculty of Medicine, University Hospital Motol, Charles University in Prague, Czech Republic

Address for correspondence: S. Adamek, MD, PhD, IIIrd Clinic of Surgery, 1st LF UK a FN Motol, V Uvalu 84, CZ-150 06 Praha 5 - Motol, Czech Republic.

Phone: +420.224 .438040$

Acknowledgement: Supported in part by grants No. VZ MSM 0021620849 and VZ MSM 0021620820. the drug or its good tolerability is not predictable for individual patients at the moment. Previously it was assumed that two-thirds of observed inter-individual cases of variability in response to opioids are due to genetic predispositions. There are several candidate genes whose polymorphisms may provide a background for the observed variability in drug response. One of them is MDR1 gene coding for P-glycoprotein responsible for efflux transport of many drugs and xenobiotics. It is highly expressed in all biological barriers, like in blood-brain barrier, testicular barrier or intestinal wall. The relationship between morphine consumption, pain scores and adverse effects in patients with different MDR1 genotypes has been studied several times. Patients carrying the wild-type allele in polymorphism of G2677T/A have a lower risk of moderate or severe drowsiness, confusion or hallucinations in comparison with patients, who carry the T allele (1). The aim of our prospective study was to evaluate the therapeutic efficacy of the piritramide in patients after the removal of parathyroid glands in relation to MDR1 genotype.

\section{Materials and methods}

A total of 56 patients with primary hyperparathyroidism, indicated for surgical removal of the parathyroid glands, have been enrolled into this prospective study. All patients signed the in- 
Tab. 1. Demographic and surgical data of patients. In MDR1 genotype groups, data are presented as mean \pm S.D. or number of patients. There were no significant differences between groups.

\begin{tabular}{lcc}
\hline & $2677 \mathrm{GG}$ & $2677 \mathrm{GT}+2677 \mathrm{TT}$ \\
\hline $\mathrm{N}$ & 15 & 41 \\
Age (years) & $49.40 \pm 7.07$ & $52.41 \pm 16.71$ \\
$\mathrm{BMI}(\mathrm{kg} / \mathrm{m} 2)$ & $26.10 \pm 3.32$ & $25.86 \pm 4.71$ \\
Gender (male/female) & $4 / 11$ & $15 / 26$ \\
Length of operation (min) & $48.5 \pm 17.0$ & $55.53 \pm 22.19$ \\
Length of anesthesia (min) & $76.42 \pm 22.39$ & $74.90 \pm 19.26$ \\
\hline
\end{tabular}

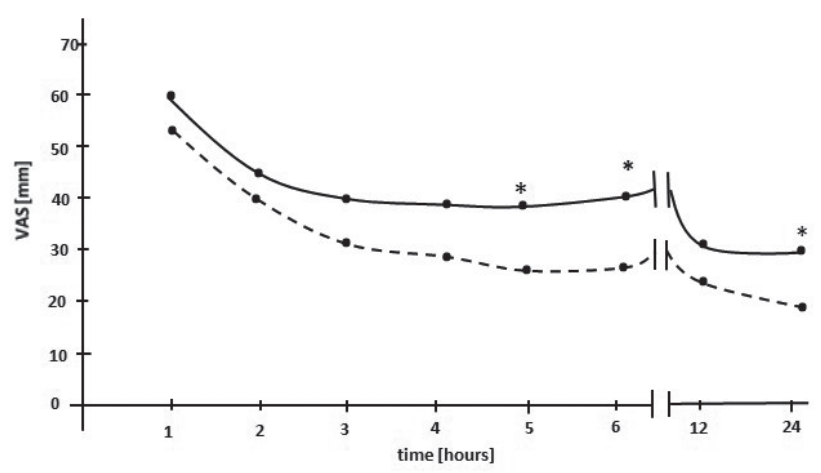

Fig. 1. Mean pain intensity rated by VAS in selected time intervals of genotype 2677GG (dashed line) and genotype 2677TT and 2677GT (solid line). * Significant differences $(p<0.05)$.

formed consent before participation. The study was approved by the local Ethics Committee and conducted in accordance with the Declaration of Helsinki. Reasons for exclusion from the study were (i) severe liver and kidney disease, (ii) depression, (iii) anxiety, (iv) epilepsy (v) opioid intolerance, and (vi) administration of non-steroidal anti-inflammatory analgesics and/or opiates one week before the surgery. Blood collection for DNA isolation was performed during the pre-operative biochemistry examination. All patients underwent surgery under general anaesthesia, based on a combination of propofol and sufentanil. For premedication, diazepam was administered per os. After returning from surgery, piritramide (Dipidolor $15 \mathrm{mg}$. inj. Sol. Janssen-Cilag Ltd) was the only administered analgesic drug throughout the study. The drug was given intramuscularly on demand at a dosage of $15 \mathrm{mg}$ for 1 application. Patients with initial pain less than $40 \mathrm{~mm}$ have not been included into this study. Pain intensity was assessed using visual analogue scale (VAS 0-100 mm) at 1, 2, 3, 4, 5, 6, 12 and 24 hours after the surgery. Differences between pre medication and post medication values of pain intensity were calculated for each scheduled and for each pain measurement. These values were used to calculate the sum of pain intensity differences (SPID), maximal pain difference and number of responders with more than $50 \%$ reduction in pain on
VAS as compared to baseline (2). In addition, patients' satisfaction with pain management and appearance of adverse drug reactions (nausea, cephalea, vomiting, sedation and vertigo) were evaluated.

Samples of peripheral venous blood were collected in tubes containing K2EDTA. The samples were immediately frozen and stored at $-20{ }^{\circ} \mathrm{C}$ until further processing. DNA was subsequently isolated using QIAmp Blood Mini Kit. Genotyping for G2677T /A polymorphism in MDR1 gene was performed by PCR-RFLP analysis, as published previously (3). Technicians responsible for genotyping were blinded to patients' clinical outcome. Analgesic consumption, pain intensity as well as piritramide medication were compared between the MDR1 genotype groups. Student's t-test (demographic data) and Mann-Whitney tests were used for statistical evaluation of differences between the two groups and $\chi^{2}$ test was used to compare the number of responders and non-responders. Differences were considered statistically significant for $\mathrm{p}<0.05$.

\section{Results}

There were fifteen patients carrying genotype $2677 \mathrm{GG}$ and 41 subjects were heterozygous or homozygous carriers of $2677 \mathrm{~T}$ allele. Heterozygous genotype of $2677 \mathrm{GA}$ was found in 3 subjects only, while no homozygous carrier of 2677A allele was detected. Therefore, the impact of 2677A allele on efficacy and safety parameters was not evaluated. There were no major complications related to surgery per se in the studied group. Demographic characteristics as well as the length or duration of anaesthesia were not significantly different between the genotype groups (Tab. 1). The average pain intensity assessed using VAS within 24 hours after surgery in both genotype groups is depicted in Figure 1. Pain intensity decrease was more pronounced in the $2677 \mathrm{GG}$ group. The values differed significantly between groups 5, 6, and 24 hours after surgery. Both maximal pain difference and SPID were significantly higher in patients with wild type of $2677 \mathrm{GG}$ genotype in comparison with the carriers of $2677 \mathrm{~T}$ allele (Tab. 2). In group of patients with wild type of $2677 \mathrm{GG}$ genotype, there were $80 \%$ of responders with a more than $50 \%$ reduction in VAS, as compared to baseline, while in group with carriers of $2677 \mathrm{~T}$ allele, there were only $39 \%$ of responders $\left(\chi^{2}=5.83 ; p=0.016\right)$. Furthermore, total consumption of piritramide, which was dosed on demand was lower in comparison with the variant-allele carrying group ( $\mathrm{p}=$ 0.008 ). Significantly more patients in the wild-type group were satisfied with the postoperative pain treatment in comparison with the variant-allele group $\left(\chi^{2}=6.49 ; p=0.0109\right)$.

Total incidence of adverse drug reactions was observed in $40 \%$ of patients with wild type of $2677 \mathrm{GG}$ genotype in comparison with $83 \%$ in the group carrying the variant allele $\left(\chi^{2}=7.92 ; p=0.005\right)$

Tab. 2. Comparison of pain characteristics in MDR1 genotype group over 24 hours postoperative period. Data are presented as mean \pm S.D.

\begin{tabular}{lcc}
\hline & $2677 \mathrm{GG}$ & $2677 \mathrm{GT}+2677 \mathrm{TT}$ \\
\hline Initial pain intensity VAS (mm) & $54.0 \pm 13.5$ & $60.9 \pm 17.0$ \\
Maximal pain difference VAS (mm) & $30.6 \pm 24.9$ & $19.5 \pm 25.5$ \\
SPID (mm) & $209.33 \pm 95.80$ & $147.07 \pm 91.38$ \\
The total amount of piritramide injections & $1.67 \pm 0.72$ & 0.023 \\
\hline
\end{tabular}

VAS - visual analogue scale, SPID - sum of pain intensity difference 


\section{Discussion}

Although there are no drug-specific data on piritramide in respect of interaction with P-glycoprotein, a number of opioids, including morfine, loperamide and fentanyl have been identified as substrates for this efflux transporter (4). Several tens of single-nucleotide polymorphisms (SNPs) have been reported in the $A B C B 1$ gene while some of these variants affect the expression and function of P-gp (5). There is a considerable controversy in respect of the significance of $2677 \mathrm{G} / \mathrm{TA}$ polymorphism, pharmacokinetics of drugs or their clinical effects. It has been shown that the $2677 \mathrm{GG}$ genotype is linked with higher fexofenadine concentrations and AUC in comparison with the variant genotypes, while preclinical data suggest lower expression of P-glycoprotein linked to the variant allele (6). A variant of 2677A allele of MDR1 gene affects the fexofenadine disposition (7). We have not studied the pharmacokinetics of piritramide in relation to $2677 \mathrm{G} / \mathrm{TA}$ polymorphism but the results of clinical efficacy suggest a better pain control in the subgroup of patients carrying the wild-type genotype of 2677 GG. These subjects consumed a smaller amount of drugs in the postoperative period while reporting significantly better maximal pain intensity, sum of pain intensity difference, and increased subjective satisfaction with the treatment. The mean values of VAS difference over 24 hours following surgery were also lower in comparison with subjects having variant alleles in the gene. We may speculate that these findings are due to increased piritramide concentrations at the site of action. This rationale would be in parallel with the pharmacokinetic observations for fexofenadine. Lower consumption of piritramide in wild-type patients probably resulted in a decreased frequency of side effects in comparison to patients with variant genotypes.

\section{Conclusion}

We observed a better analgesic effect of piritramide in the wild-type patient group with $2677 \mathrm{GG}$ genotype compared with patients carrying a variant allele. Lower total consumption of the drug in the $2677 \mathrm{GG}$ group was connected with a decreased incidence of side effects and better subjective satisfaction with postoperative pain control. It is expected that MDR gene genotyping is going to be of importance for future progress and quality of post-operative period.

\section{References}

1. Rakvag TT, Ross JR, Sato H, Skorpen F, Kaasa S, Klepstad P. Genetic variation in the Catechol-O-Methyltransferase (COMT) gene and morphine requirements in cancer patients with pain. Mol Pain 2008; 4: 64-76.

2. Moore A, Moore O, McQuay H, Gavaghan D. Deriving dichotomous outcome measures from continuous data in randomised controlled trials of analgesics: Use of pain intensity and visual analogue scales. Pain 1997; 69 (3): 311-315.

3. Pechandova K, Buzkova H, Slanar O, Perlik F. Polymorphisms of the MDR1 gene in the Czech population. Folia Biol 2006; 52 (6): 184-189.

4. Somogyi AA, Barratt DT, Coller JK. Pharmacogenetics of opioids. Clin Pharmacol Ther 2007; 81 (3): 429-444.

5. Fromm MF. The influence of MDR1 polymorphisms on P-glycoprotein expression and function in humans. Adv Drug Deliv Rev 2002; 54 (10): 1295-1310.

6. Yi SY, Hong KS, Lim HS, Chung J, Oh DS, Kim JR, Jung HR, et al. A variant 2677A allele of the MDR1 gene affects fexofenadine disposition. Clin Pharmacol Ther 2004; 76 (5): 418-427.

7. Song P, Lamba JK, Zhang L, Schuetz E, Shukla N, Meibohm B, Yates CR. G2677T and C3435T genotype and haplotype are associated with hepatic ABCB1 (MDR1) expression. J Clin Pharmacol 2006; 46 (3): 373-379.

Received January 18, 2011. Accepted December 17, 2012. 